\title{
NUMERICAL ASPECTS OF NONLINEAR SCHRÖDINGER EQUATIONS IN THE PRESENCE OF CAUSTICS
}

\author{
RÉMI CARLES AND LAURENT GOSSE
}

\begin{abstract}
The aim of this text is to develop on the asymptotics of some 1-D nonlinear Schrödinger equations from both the theoretical and the numerical perspectives, when a caustic is formed. We review rigorous results in the field and give some heuristics in cases where justification is still needed. The scattering operator theory is recalled. Numerical experiments are carried out on the focus point singularity for which several results have been proven rigorously. Furthermore, the scattering operator is numerically studied. Finally, experiments on the cusp caustic are displayed, and similarities with the focus point are discussed.
\end{abstract}

\section{INTRODUCTION}

We present a numerical study of the semi-classical solutions to the following nonlinear Schrödinger equations with $\varepsilon \ll 1$,

(1.1) $i \varepsilon \partial_{t} \mathrm{u}^{\varepsilon}+\frac{\varepsilon^{2}}{2} \Delta \mathrm{u}^{\varepsilon}=\left|\mathrm{u}^{\varepsilon}\right|^{2 \sigma} \mathrm{u}^{\varepsilon}, \quad(t, x) \in \mathbb{R}_{+} \times \mathbb{R}^{n} \quad ; \quad \mathrm{u}_{\mid t=0}^{\varepsilon}=\varepsilon^{p} f(x) e^{i \phi_{0}(x) / \varepsilon}$,

when a caustic (a point or a cusp) is formed, that is to say, beyond breakup time. Since the nonlinearity is homogeneous, the change of unknown function $u^{\varepsilon}=\varepsilon^{p} \mathbf{u}^{\varepsilon}$ shows that (1.1) is equivalent to:

$$
i \varepsilon \partial_{t} u^{\varepsilon}+\frac{\varepsilon^{2}}{2} \Delta u^{\varepsilon}=\varepsilon^{2 \sigma p}\left|u^{\varepsilon}\right|^{2 \sigma} u^{\varepsilon} \quad ; \quad u_{\mid t=0}^{\varepsilon}=f(x) e^{i \phi_{0}(x) / \varepsilon},
$$

so that we can always consider initial data of order $\mathcal{O}(1)$.

There are several motivations to study the behavior of (1.2) when a caustic is formed. First, on a purely academic level, we recall that the description of the caustic crossing is complete in the case of linear equations; see [17. For nonlinear equations, very interesting formal computations were proposed in 25] (we recall the main idea in Section 2 below). For dissipative nonlinear wave equations, Joly, Métivier and Rauch [26, 27] have proved that the amplification of the wave near the caustic can ignite the dissipation phenomenon in such a way that the oscillations (that carry highest energy) are absorbed. The above nonlinear Schrödinger equation is the simplest model of a conservative, nonlinear equation. The mass and the energy of the solution are independent of time (see (2.7) below). Therefore, different

2000 Mathematics Subject Classification. 35B33, 35P25, 35Q55, 65T50, 81Q20.

Key words and phrases. Nonlinear Schrödinger equation, time-splitting scheme, Fourier scheme, WKB expansion, caustics.

This work was partially supported by o Centro de Matemática e Aplicações Fundamentais (Lisbon), funded by FCT as contract POCTI-ISFL-1-209, and by the Austrian Ministry of Science via its grant for the Wolfgang Pauli Institute and by the Austrian Science Foundation (FWF) via the START Project (Y-137-TEC).. 
nonlinear mechanisms are expected. We recall in Section 2 some results that have been established rigorously, and give heuristic arguments to extend these results. This serves as a guideline for the numerical experiments proposed after.

Second, (1.2) may be considered as a simplified model for Bose-Einstein condensation, which may be modeled (see e.g. [16, 32]) by:

$$
i \varepsilon \partial_{t} u^{\varepsilon}+\frac{\varepsilon^{2}}{2} \Delta u^{\varepsilon}=\omega^{2} \frac{|x|^{2}}{2} u^{\varepsilon}+\varepsilon^{2}\left|u^{\varepsilon}\right|^{2 \sigma} u^{\varepsilon},
$$

with $\sigma=2$ if $n=1$, and $\sigma=1$ if $n=2$ or 3 . The power $\varepsilon^{2}$ in front of the nonlinearity depends on the régime considered, and in particular on the respective scales of different parameters (see e.g. [7] and references therein). The role of the harmonic potential $|x|^{2}$ is to model a magnetic trap. In the semi-classical limit $\varepsilon \rightarrow 0$ for the linear equation, this potential causes focusing at the origin for solutions whose data are independent of $\varepsilon$. This is to be compared with the case of (1.2) with initial quadratic oscillations as considered below: the initial quadratic oscillations force the solution to concentrate at one point in the limit $\varepsilon \rightarrow 0$. The parallel between (1.2) and (1.3) was extended and justified in 10 for these nonlinear equations.

From both points of view, when a caustic point is formed, the caustic crossing may be described in terms of the scattering operator associated to

$$
i \partial_{t} \psi+\frac{1}{2} \Delta \psi=|\psi|^{2 \sigma} \psi
$$

This aspect is recalled in Section 2. For this reason, we also pay a particular attention to this operator, independently of the above semi-classical limit. Note that besides the existence of this operator, very few of its properties (dynamical, for instance) are known.

In this paper, we always assume $2 \sigma p \geq 1$ : one of the reasons is that when $0 \leq 2 \sigma p<1$, instability occurs, see 12 , 13. Suppose for instance that $u^{\varepsilon}$ solves (1.2), and that $\widetilde{u}^{\varepsilon}$ solves (1.2), where $f$ replaced by $\left(1+\delta^{\varepsilon}\right) f$, where $\delta^{\varepsilon}$ is a sequence of real numbers going to zero as $\varepsilon \rightarrow 0$. Then there are some choices of $\delta^{\varepsilon}$ for which

$$
\liminf _{\varepsilon \rightarrow 0}\left\|u^{\varepsilon}\left(t^{\varepsilon}\right)-\widetilde{u}^{\varepsilon}\left(t^{\varepsilon}\right)\right\|_{L^{2}}>0
$$

for some sequence of time $t^{\varepsilon} \rightarrow 0$ (see [12], and [7] for a similar phenomenon with different initial data). Therefore, producing reliable numerical tests in the case $0 \leq 2 \sigma p<1$ (which is super-critical as far as WKB analysis is concerned [12, 13]) seems to be a very delicate issue, that we leave out in the present paper.

The rest of this paper is structured as follows. In Section 2, we recall the general approach of WKB analysis for the Schrödinger equation, the arguments of [25], and the rigorous results available for the semi-classical limit of (1.2) when a caustic reduced to a point is formed. We then recall the definition of the scattering operator. We also give heuristic arguments to tackle the case of a "supercritical focal point", and to guess what the critical indices are when a cusp caustic is formed, instead of a focal point. In Section 3, we present the different strategies that have been followed in the literature to study numerically the semi-classical limit for nonlinear Schrödinger equations. Numerical experiments on the semi-classical limit for (1.2) in the presence of a focal point appear in Section 4 , and the scattering operator is simulated in Section 5. We present the numerical experiments of the semi-classical 
limit for (1.2) in the presence of a cusp caustic in Section 6, and make conclusive remarks in Section 0 .

\section{Analytical approach}

2.1. Semi-classical limit of the free Schrödinger equation. Consider the initial value problem, for $(t, x) \in \mathbb{R}_{+} \times \mathbb{R}^{n}$ :

$$
i \varepsilon \partial_{t} v^{\varepsilon}+\frac{\varepsilon^{2}}{2} \Delta v^{\varepsilon}=0 \quad ; \quad v_{\mid t=0}^{\varepsilon}=f(x) e^{i \phi_{0}(x) / \varepsilon} .
$$

The aim of WKB methods is to describe the asymptotic behavior of $v^{\varepsilon}$ as $\varepsilon \rightarrow 0$. For instance, $\varepsilon$ can be related to the Planck constant, and the asymptotic behavior of $v^{\varepsilon}$ is expected to yield a good description of $v^{\varepsilon}$ when $\varepsilon$ is fixed, but small compared to the other parameters. More precisely, seek $v^{\varepsilon}$ of the form

$$
v^{\varepsilon}(t, x) \sim e^{i \phi(t, x) / \varepsilon}\left(a_{0}(t, x)+\varepsilon a_{1}(t, x)+\ldots\right) \quad \text { as } \varepsilon \rightarrow 0 .
$$

Plugging this expansion into (2.1) and canceling the $\mathcal{O}\left(\varepsilon^{0}\right)$ term, we see that the phase $\phi$ must solve the eikonal equation:

$$
\partial_{t} \phi+\frac{1}{2}|\nabla \phi|^{2}=0 \quad ; \quad \phi_{\mid t=0}=\phi_{0} .
$$

To cancel the $\mathcal{O}\left(\varepsilon^{1}\right)$ term, the leading order amplitude solves the transport equation:

$$
\partial_{t} a_{0}+\nabla \phi \cdot \nabla a_{0}+\frac{1}{2} a_{0} \Delta \phi=0 \quad ; \quad a_{0 \mid t=0}=f .
$$

The eikonal equation (2.3) is solved thanks to Hamilton-Jacobi theory ${ }^{1}: \phi$ is constructed locally in space and time (see e.g. 13] for a discussion on this aspect). Even if $\phi_{0}$ is smooth, $\phi$ develops singularities in finite time in general: the locus where $\phi$ is singular is called caustic (see e.g. the second volume of 24]). When $\phi$ becomes singular, all the terms $a_{0}, a_{1}, \ldots$ may become singular as well. One easily observes that (2.4) admits a "divergence form": $\partial_{t}\left|a_{0}\right|^{2}+\nabla \cdot\left(\left|a_{0}\right|^{2} \nabla \phi\right)=0$. To illustrate this general discussion, we consider two examples that will organize the rest of this paper.

Example (Quadratic phase). Let $\phi_{0}(x)=-\frac{|x|^{2}}{2}$. Then (2.3) and (2.4) can be solved explicitly:

$$
\phi(t, x)=\frac{|x|^{2}}{2(t-1)} \quad ; \quad a(t, x)=\frac{1}{(1-t)^{n / 2}} f\left(\frac{x}{1-t}\right) .
$$

This shows that as $t \rightarrow 1, \phi$ and $a$ become singular: the wave $u^{\varepsilon}$ focuses at the origin. This example can be viewed as the smooth counterpart of the Cauchy problem

$$
i \partial_{t} \psi+\frac{1}{2} \Delta \psi=0 \quad ; \quad \psi_{\mid t=0}=e^{-i \frac{|x|^{2}}{2}} .
$$

Fourier analysis shows that $\psi_{\mid t=1}=\delta$, the Dirac measure at the origin.

Of course, the solution of (2.1) can be represented as an oscillatory integral:

$$
v^{\varepsilon}(t, x)=\frac{1}{(2 i \pi t)^{n / 2}} \int e^{i \frac{|x-y|^{2}}{2 \varepsilon t}+i \frac{\phi_{0}(y)}{\varepsilon}} f(y) d y .
$$

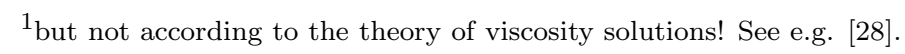


The caustic set is exactly the locus where the critical points for the phase

$$
\Phi_{t, x}(y)=\frac{|x-y|^{2}}{2 t}+\phi_{0}(y)
$$

are degenerate. Outside the caustic, an approximation of $v^{\varepsilon}$ is given by the stationary phase theorem (that we recalled as simply as possible in [22]). This leads us to the second example we shall consider numerically:

Example (Cusp). Let $n=1$ and $\phi_{0}(x)=\cos x$. The set of degenerate critical points for $\Phi_{t, x}(y)$ (caustic) is given implicitly by:

$$
\mathcal{C}=\left\{(t, x) \in \mathbb{R}_{+} \times \mathbb{R} ; \exists y \in \mathbb{R}, \frac{y-x}{t}=\sin y, \text { and } \frac{1}{t}=\cos y\right\} .
$$

As soon as $t \geq 1$, a caustic is formed (see Figure 2 in [23]).

When considering the asymptotic behavior of $u^{\varepsilon}$ beyond the caustic, two main features must be considered: the creation of other phases ${ }^{2}$, and the Maslov index (see [17 for more general linear equations). In the case of a focal point, the first aspect does not exist: there is no creation of phase, and one phase is enough to describe $v^{\varepsilon}$ past the focal point $(t, x)=(1,0)$. One can prove easily the following result:

Lemma 2.1. Let $n \geq 1$ and $f \in \mathcal{S}\left(\mathbb{R}^{n} ; \mathbb{C}\right)$. If $\phi_{0}(x)=-|x|^{2} / 2$, then the asymptotic behavior (in $L^{2}\left(\mathbb{R}^{n}\right)$ ) of the solution $v^{\varepsilon}$ to $(2.1)$ is given by:

$$
v^{\varepsilon}(t, x) \underset{\varepsilon \rightarrow 0}{\sim}\left\{\begin{aligned}
\frac{e^{i|x|^{2} /(2 \varepsilon(t-1))}}{(1-t)^{n / 2}} f\left(\frac{x}{1-t}\right) & \text { if } t<1, \\
e^{-i n \frac{\pi}{2}} \frac{e^{i|x|^{2} /(2 \varepsilon(t-1))}}{(t-1)^{n / 2}} f\left(\frac{x}{1-t}\right) & \text { if } t>1 .
\end{aligned}\right.
$$

In this example, the Maslov index is $-n \pi / 2$. In the case of the cusp, three phases must be considered to describe the asymptotic behavior of $v^{\varepsilon}$ beyond the caustic (see e.g. [23, 22]).

For future discussion on the numerical results, we state the following more precise result, which follows from the stationary phase theorem:

Lemma 2.2. Let $n \geq 1$ and $f \in \mathcal{S}\left(\mathbb{R}^{n} ; \mathbb{C}\right)$. If $\phi_{0}(x)=-|x|^{2} / 2$, then the asymptotic behavior of the solution $v^{\varepsilon}$ to (2.1) at time $t=2$ is given by:

$$
v^{\varepsilon}(2, x)=e^{-i n \frac{\pi}{2}} e^{i|x|^{2} /(2 \varepsilon)} f(-x)+O(\varepsilon) \quad \text { in } L^{2} \cap L^{\infty}\left(\mathbb{R}^{n}\right) .
$$

2.2. Caustics in the nonlinear case: heuristics. Consider now the perturbation of (2.1) with a nonlinear term:

$$
i \varepsilon \partial_{t} u^{\varepsilon}+\frac{\varepsilon^{2}}{2} \Delta u^{\varepsilon}=\varepsilon^{\alpha}\left|u^{\varepsilon}\right|^{2 \sigma} u^{\varepsilon} \quad ; \quad u_{\mid t=0}^{\varepsilon}=f(x) e^{i \phi_{0}(x) / \varepsilon} .
$$

The sign of the nonlinearity is chosen so that no finite time blow-up occurs. The following two important quantities are formally independent of time:

$$
\begin{aligned}
& \text { Mass: }\left\|u^{\varepsilon}(t)\right\|_{L^{2}}=\text { Const. }=\|f\|_{L^{2}} . \\
& \text { Energy: } E^{\varepsilon}(t):=\frac{1}{2}\left\|\varepsilon \nabla u^{\varepsilon}(t)\right\|_{L^{2}}^{2}+\frac{\varepsilon^{\alpha}}{\sigma+1}\left\|u^{\varepsilon}(t)\right\|_{L^{2 \sigma+2}}^{2 \sigma+2}=E^{\varepsilon}(0) .
\end{aligned}
$$

\footnotetext{
2 in our mind, phases are always associated to oscillations whose period depends on $\varepsilon$, and goes to infinity as $\varepsilon \rightarrow 0$ - rapid oscillations. The wavelength may be proportional to $\varepsilon$, or, say, to $\sqrt{\varepsilon}$.
} 
We refer to 14 for a justification. Fix the power $2 \sigma>0$ of the nonlinearity, and consider different values for $\alpha$. Two notions of criticality arise: for the WKB methods on the one hand, and for the caustic crossing on the other hand. This discussion is presented in 25] for conservation laws, and we summarize it in the case of (2.6). Plugging an expansion of the form (2.2) into (2.6), we see that the value $\alpha=1$ is critical for the WKB methods: if $\alpha>1$, then the nonlinearity does not affect the transport equation (2.4) ("linear propagation"), while if $\alpha=1$, then the nonlinearity appears in the right hand side of (2.4) ("nonlinear propagation"). Recall that in this paper, we always assume $\alpha \geq 1$. Therefore, the eikonal equation (2.3) is not altered: the geometry of the propagation remains the same as in the linear WKB approach, and we have to face the same caustic sets. The idea presented in 25] consists in saying that according to the geometry of the caustic, different notions of criticality exist, as far as $\alpha$ is concerned, near the caustic. In the linear setting (2.1), the influence of the caustic is relevant only in a neighborhood of this set (essentially, in a boundary layer whose size depends on $\varepsilon$ and the geometry of $\mathcal{C})$. View the nonlinearity in (2.6) as a potential, and assume that the nonlinear effects are negligible near the caustic: then $u^{\varepsilon} \sim v^{\varepsilon}$ near $\mathcal{C}$. View the term $\varepsilon^{\alpha}\left|u^{\varepsilon}\right|^{2 \sigma}$ as a (nonlinear) potential. The average nonlinear effect near $\mathcal{C}$ is expected to be:

$$
\varepsilon^{-1} \int_{\mathcal{C}^{\varepsilon}} \varepsilon^{\alpha}\left|u^{\varepsilon}\right|^{2 \sigma} \sim \varepsilon^{-1} \int_{\mathcal{C}^{\varepsilon}} \varepsilon^{\alpha}\left|v^{\varepsilon}\right|^{2 \sigma},
$$

where $\mathcal{C}^{\varepsilon}$ is the region where caustic effects are relevant, and the factor $\varepsilon^{-1}$ is due to the integration in time (recall that there is an $\varepsilon$ in front of the time derivative in (2.6)). The idea of this heuristic argument is that when the nonlinear effects are negligible near $\mathcal{C}$ (in the sense that the uniform norm of $u^{\varepsilon}-v^{\varepsilon}$ is small compared to that of $v^{\varepsilon}$ near $\mathcal{C}^{\varepsilon}$ ), the above approximation should be valid. On the other hand, it is expected that it ceases to be valid precisely when nonlinear effects can no longer be neglected near the caustic: $u^{\varepsilon}-v^{\varepsilon}$ is of the same order of magnitude as $v^{\varepsilon}$ in $L^{\infty}\left(\mathcal{C}^{\varepsilon}\right)$, or even larger.

Practically, assume that in the linear case, $v^{\varepsilon}$ has an amplitude $\varepsilon^{-\ell}$ in a boundary layer of size $\varepsilon^{k}$; then the above quantity is

$$
\varepsilon^{-1} \int_{\mathcal{C}^{\varepsilon}} \varepsilon^{\alpha}\left|v^{\varepsilon}\right|^{2 \sigma} \sim \varepsilon^{-1} \varepsilon^{\alpha}\left|\varepsilon^{-\ell}\right|^{2 \sigma} \varepsilon^{k} .
$$

The value $\alpha$ is then critical when the above cumulated effects are not negligible:

$$
\alpha_{c}=1+2 \ell \sigma-k .
$$

When $\alpha>\alpha_{c}$, the nonlinear effects are expected to be negligible near the caustic: resuming the terminology of [25], we speak of "linear caustic". The case $\alpha=\alpha_{c}$ is called "nonlinear caustic". To conclude this paragraph, we examine this approach in the case of our two examples. In the case of a focal point, we have $k=1$ and $\ell=n / 2$. This leads us to the value:

$$
\alpha_{c}(\text { focal point })=n \sigma .
$$

In the case of the cusp in dimension one, we have $k=2 / 3$ and $\ell=1 / 3$ (which can be viewed thanks to the Airy function and its asymptotic expansion, see e.g. 17, 24, 25 or 29), which yields:

$$
\alpha_{c}(\operatorname{cusp} \text { in } 1 \mathrm{D})=\frac{2 \sigma+1}{3} .
$$


One aspect of the numerical experiments presented below is to test this notion of criticality in those two examples.

2.3. Justification for a focal point, and more heuristics. In this paragraph, we assume $\phi_{0}(x)=-|x|^{2} / 2$. A complete justification of the above discussion is available [8]:

\begin{tabular}{c|c|c} 
& $\alpha>n \sigma$ & $\alpha=n \sigma$ \\
\hline$\alpha>1$ & $\begin{array}{c}\text { Linear caustic, } \\
\text { linear propagation }\end{array}$ & $\begin{array}{c}\text { Nonlinear caustic, } \\
\text { linear propagation }\end{array}$ \\
\hline$\alpha=1$ & $\begin{array}{c}\text { Linear caustic, } \\
\text { nonlinear propagation }\end{array}$ & $\begin{array}{c}\text { Nonlinear caustic, } \\
\text { nonlinear propagation }\end{array}$
\end{tabular}

Consider $t \in[0,2]$, which includes the caustic crossing. The above tables means:

- If $\alpha>\max (1, n \sigma)$, then $u^{\varepsilon}$ can be approximated by $v^{\varepsilon}$ for $t \in[0,2]$.

- If $\alpha=1>n \sigma$, then the nonlinearity is negligible near the focal point, but not away from it.

- If $\alpha=n \sigma>1$, then nonlinear effects are relevant near the focal point, and only near the focal point.

- If $\alpha=n \sigma=1$, then the nonlinearity is never negligible.

We give some precisions in some cases of interest for the numerics presented below.

In the one-dimensional case $n=1$, the following pointwise estimate is proved in 8] when $\alpha>\max (1, \sigma)$ or $\alpha=\sigma>1$ :

$$
\left|u^{\varepsilon}(t, x)\right| \leq \frac{C}{\sqrt{|t-1|+\varepsilon}}
$$

Setting $w^{\varepsilon}=u^{\varepsilon}-v^{\varepsilon}$, we see that

$$
i \varepsilon \partial_{t} w^{\varepsilon}+\frac{\varepsilon^{2}}{2} \partial_{x}^{2} w^{\varepsilon}=\varepsilon^{\alpha}\left|u^{\varepsilon}\right|^{2 \sigma} u^{\varepsilon} \quad ; \quad w_{\mid t=0}^{\varepsilon}=0 .
$$

The usual energy estimate yields, for $t \geq 0$ :

$$
\left\|w^{\varepsilon}(t)\right\|_{L^{2}} \leq \varepsilon^{-1} \int_{0}^{t} \varepsilon^{\alpha}\left\|\left|u^{\varepsilon}(\tau)\right|^{2 \sigma} u^{\varepsilon}(\tau)\right\|_{L^{2}} d \tau .
$$

Using (2.8), we infer, for $t \in[0,2]$ :

$$
\left\|w^{\varepsilon}(t)\right\|_{L^{2}} \lesssim \varepsilon^{\alpha-1}\left(\int_{\{|\tau-1|>\varepsilon\} \cap\{\tau \in[0,2]\}} \frac{d \tau}{|\tau-1|^{\sigma}}+\int_{|\tau-1| \leq \varepsilon} \frac{d \tau}{\varepsilon^{\sigma}}\right) \lesssim \varepsilon^{\alpha-\sigma}
$$

Using the operator $\varepsilon \partial_{x}$ and $x / \varepsilon+i(t-1) \partial_{x}$, and Gagliardo-Nirenberg inequalities as in [8], we find:

Lemma 2.3. Let $n=1, \alpha>\max (1, \sigma), f \in \mathcal{S}\left(\mathbb{R}^{n} ; \mathbb{C}\right)$, and $\phi_{0}(x)=-|x|^{2} / 2$. Then we have, for the solutions of (2.1) and (2.6):

$\sup _{0 \leq t \leq 2}\left\|u^{\varepsilon}(t)-v^{\varepsilon}(t)\right\|_{L^{2}} \leq C \varepsilon^{\alpha-\sigma} \quad ; \quad\left\|u^{\varepsilon}(t)-v^{\varepsilon}(t)\right\|_{L^{\infty}} \leq C \frac{\varepsilon^{\alpha-\sigma}}{\sqrt{|t-1|+\varepsilon}}, \quad t \in[0,2]$.

In particular, Lemma 2.3 implies, at time $t=2$ :

$$
u^{\varepsilon}(2, x)=e^{-i n \frac{\pi}{2}} e^{i|x|^{2} /(2 \varepsilon)} f(-x)+O\left(\varepsilon^{\min (1, \alpha-\sigma)}\right) \quad \text { in } L^{2} \cap L^{\infty}(\mathbb{R}) .
$$


(The above result is true also when $\alpha=\sigma>1$, but becomes far less interesting.) We now explain the critical case $\alpha=n \sigma>1$. The nonlinear effects near the focal point are described in terms of the scattering operator associated to the nonlinear Schrödinger equation. We rapidly present this operator $S$ in Section 2.4. We have then:

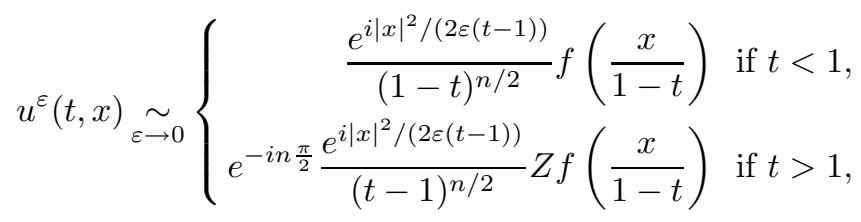

where $Z=\mathcal{F} \circ S \circ \mathcal{F}^{-1}$ is the conjugate of $S$ by the Fourier transform (see [8]),

$$
\mathcal{F} \varphi(\xi)=\frac{1}{(2 i \pi)^{n / 2}} \int e^{-i x \cdot \xi} \varphi(x) d x .
$$

(Since $S$ is a nonlinear operator, the normalization of the Fourier transform is an important detail.)

To conclude this paragraph, we give a few hints of what happens or may happen when the propagation is linear, and the caustic is super-critical, that is $1<\alpha<n \sigma$. First, the conservation of mass and energy seem to rule out the possibility of a concentration of the form

$$
u^{\varepsilon}(1, x) \sim \frac{1}{\varepsilon^{n / 2}} \varphi\left(\frac{x}{\varepsilon}\right),
$$

for some function $\varphi$ independent of $\varepsilon$. The above relation holds for $v^{\varepsilon}$ and $u^{\varepsilon}$ when $\alpha>\max (1, n \sigma)$, with $\varphi=\mathcal{F} f$ (for $v^{\varepsilon}$, this is obvious from (2.5)). When $\alpha=n \sigma>1$ (linear propagation and nonlinear focal point), the above relation still holds, with a different profile $\varphi$ (see [8]). Now we see that the energy is bounded as $\varepsilon \rightarrow 0$ :

$$
E^{\varepsilon}(t)=E^{\varepsilon}(0) \underset{\varepsilon \rightarrow 0}{\sim} \frac{1}{2}\|x f\|_{L^{2}}^{2} .
$$

Plugging a concentrating profile as in (2.9) in the second term of the energy would yield, thanks to the conservation of mass:

$$
\varepsilon^{\alpha}\left\|u^{\varepsilon}(1)\right\|_{L^{2 \sigma+2}}^{2 \sigma+2} \approx \varepsilon^{\alpha-n \sigma} \underset{\varepsilon \rightarrow 0}{\longrightarrow}+\infty
$$

which is impossible since the energy is the sum of two positive terms. This suggests two possible effects: near $t=1$, the amplification of the solution (in terms of powers of $\varepsilon$ ) may be weaker than in the linear case; on the other hand, nonlinear effects near the caustic should affect the phase of the solution $u^{\varepsilon}$ in a rather strong way, causing the appearance of new frequencies. A partial justification of the last assertion may be found in [11].

Extrapolating this argument, we expect that in the supercritical case for a cusp (with $n=1: \frac{2 \sigma+1}{3}>\alpha>1$ ), new frequencies appear. If as in the linear case, three phases are necessary to describe the solution past the caustic, then the nonlinear interaction of these phases might reveal the presence of new frequencies, even on the modulus of $u^{\varepsilon}$ (see Sect. 6 for numerical tests that seem to confirm this heuristics). 
2.4. The scattering operator for NLS. To explain what the operator $S$ mentioned in the previous section is, consider the nonlinear Schrödinger equation

$$
\begin{aligned}
i \partial_{t} \psi+\frac{1}{2} \Delta \psi & =|\psi|^{2 \sigma} \psi \quad ; \quad(t, x) \in \mathbb{R} \times \mathbb{R}^{n}, \\
U(-t) \psi(t)_{\mid t=t_{0}} & =\psi_{-},
\end{aligned}
$$

where $U(t)=e^{i \frac{t}{2} \Delta}$ is the propagator of the linear equation. To construct the scattering operator, we first want to give a meaning to (2.10)-2.11) when $t_{0}=-\infty$. This means that the nonlinear effects are asymptotically negligible as $t \rightarrow-\infty$ : for instance, we expect at least

$$
\left\|U(-t) \psi(t)-\psi_{-}\right\|_{L^{2}}=\left\|\psi(t)-U(t) \psi_{-}\right\|_{L^{2}} \underset{t \rightarrow-\infty}{\longrightarrow} 0 .
$$

This gives a rigorous meaning to the relation $\psi(t, x) \sim U(t) \psi_{-}(x)$ which aims at saying that as time goes to $-\infty$, the nonlinear dynamics associated to (2.10) can be compared to the free dynamics given by $e^{i \frac{t}{2} \Delta}$.

To define a scattering operator, we want to be able to say that as $t \rightarrow+\infty$ as well, the nonlinear effect are asymptotically negligible. That is, there exists $\psi_{+} \in L^{2}\left(\mathbb{R}^{n}\right)$ such that

$$
\left\|U(-t) \psi(t)-\psi_{+}\right\|_{L^{2}}=\left\|\psi(t)-U(t) \psi_{+}\right\|_{L^{2}} \underset{t \rightarrow+\infty}{\longrightarrow} 0 .
$$

The scattering operator is then defined as $S: \psi_{-} \mapsto \psi_{+}$. Since our numerical experiments concern the one-dimensional case, we recall the existence of the scattering operator in this setting, and refer to [14, 15, 20, 21, 31] for some extensions to the multidimensional framework: define

$$
\Sigma:=H^{1} \cap \mathcal{F}\left(H^{1}\right)=\left\{f \in L^{2}\left(\mathbb{R}^{n}\right) ;\|f\|_{\Sigma}:=\|f\|_{L^{2}}+\|x f\|_{L^{2}}+\|\nabla f\|_{L^{2}}<\infty\right\} .
$$

Proposition 2.4 (Scattering theory). Let $n=1$, and assume $\sigma \geq \frac{1+\sqrt{17}}{4}(>1)$.

- For every $\psi_{-} \in \Sigma$, there exists a unique $\varphi \in \Sigma$ such that the maximal solution $\psi \in C(\mathbb{R}, \Sigma)$ to (2.10) satisfies $\psi_{\mid t=0}=\varphi$ and

$$
\left\|U(-t) \psi(t)-\psi_{-}\right\|_{\Sigma} \underset{t \rightarrow-\infty}{\longrightarrow} 0 .
$$

- For every $\varphi \in \Sigma$, there exists a unique $\psi_{+} \in \Sigma$ such that the maximal solution $\psi \in C(\mathbb{R}, \Sigma)$ to (2.10) with $\psi_{\mid t=0}=\varphi$ satisfies

$$
\left\|U(-t) \psi(t)-\psi_{+}\right\|_{\Sigma} \underset{t \rightarrow+\infty}{\longrightarrow} 0 .
$$

The scattering operator is $S: \psi_{-} \mapsto \psi_{+}$. When $\sigma>1$, the above conclusions remain, in a neighborhood of the origin. When $\sigma>1$, we also have:

- For every $\psi_{-} \in \Sigma$, there exist a unique solution $\psi \in C\left(\mathbb{R}, H^{1}\right)$ to (2.10) and a unique $\psi_{+} \in H^{1}(\mathbb{R})$ such that:

$$
\left\|U(-t) \psi(t)-\psi_{-}\right\|_{\Sigma} \underset{t \rightarrow-\infty}{\longrightarrow} 0 ; \quad\left\|U(-t) \psi(t)-\psi_{+}\right\|_{L^{2}} \underset{t \rightarrow+\infty}{\longrightarrow} 0 .
$$

When $\sigma \leq 1$, the above conclusions are false: if $\sigma=1$ for instance, and if $\psi_{-} \in L^{2}$ with $U_{0}(-t) \psi(t)-\psi_{-} \rightarrow 0$ in $L^{2}$ as $t \rightarrow-\infty$, then $\psi=\psi_{-}=0$. One cannot compare the nonlinear dynamics with the free dynamics (see 3, 33, 34, 19 ). Note that even though the scattering is proven to exist, very few of its features are known. We refer to [14] for some algebraic properties. At least, this operator is not trivial: near the origin, it is a non-trivial perturbation of the identity (see [9]). 


\section{Numerical approximation of Semi-Classical Schrödinger EQUations}

Hereafter we restrict our discussion to the one-dimensional case, that is to say $n=1$ in all the preceding considerations.

3.1. Rigorous results for general time-splitting schemes. It is interesting to notice that in the numerical literature, the nonlinear equation $(2.6)$ is treated exactly the same way the linear one (2.1) would be in the presence of a potential $V$ on its right-hand side. The strategy is called time-splitting, in its first or second order version (Lie or Strang splitting, see e.g. [ 4 ) where one alternates every time step $\Delta t>0$ between the solving of the Laplace operator and the handling of the (nonlinear) differential equation. According to Section 2.4, $U$ will still stand for the free propagator, whereas we shall use $V$ as the ODE solver; Lie time-splitting algorithms generate the following type of approximation for (2.6),

$$
u^{\varepsilon}(n \Delta t, .) \simeq u_{\Delta t}^{\varepsilon}(n \Delta t, .):=[V(\Delta t) \circ U(\Delta t)]^{n} u^{\varepsilon}(t=0, .),
$$

and Strang splittings,

$u^{\varepsilon}(n \Delta t,.) \simeq \tilde{u}_{\Delta t}^{\varepsilon}(n \Delta t,):.=V(\Delta t / 2) \circ U(\Delta t)[V(\Delta t) \circ U(\Delta t)]^{n-1} V(\Delta t / 2) u^{\varepsilon}(t=0,$.$) .$

Many references exist; let us quote only [4, 30, 1, 2].

On the contrary, few rigorous convergence results are available, hence we shall mainly recall the results from [4] which quantify accurately the splitting errors assuming each time-step is performed exactly ${ }^{3}$. Under this assumption, there holds:

Proposition 3.1. (四, Theorem 4.1) For any $T>0$ and $u^{\varepsilon}(t=0,.) \in H^{2}$, there exists a constant $C$ depending on the initial data for (2.6) and $h_{0}$, such that for $\Delta t \in\left[0, h_{0}\right]$ and $n \Delta t<T$,

$$
\left\|u^{\varepsilon}(n \Delta t, .)-u_{\Delta t}^{\varepsilon}(n \Delta t, .)\right\|_{L^{2}} \leq C h_{0} .
$$

If moreover $u^{\varepsilon}(t=0,.) \in H^{4}$, then there holds under the same assumptions:

$$
\left\|u^{\varepsilon}(n \Delta t, .)-\tilde{u}_{\Delta t}^{\varepsilon}(n \Delta t, .)\right\|_{L^{2}} \leq C h_{0}^{2} .
$$

This result is concerned with splitting errors only and relies on the knowledge of the exact solution operators $U$ and $V$. In order to stick to this framework in the context of smooth solutions, it is rather natural to approximate $U$ by means of a Fourier scheme taking advantage of optimized FFT routines, as proposed in the paper [2]. Moreover, this will guarantee that the $L^{2}$ norm (Mass) of the numerical solution will be conserved up to round-off errors. Unfortunately, the Hamiltonian $E^{\varepsilon}(t)$ is generally not preserved; a method conserving both quantities exists (see the so-called MCN algorithm, page 253 of 18 ) but it wouldn't be efficient in the semiclassical regime because of the results in [30].

3.2. Specific issues with finite-difference discretizations. This is the main purpose of the paper [30] to illustrate the (surprising) fact that in semi-classical regime, usual finite-difference schemes for (2.1) can deliver very wrong approximations without any particular sign of instability in case very restrictive meshing constraints turn out to be bypassed. This can be quite easily checked through the location of caustics, for instance. The analysis of those standard schemes has been carried out by means of Wigner measures, so the conclusions hold essentially for the quadratic observables coming out of the wave function itself.

\footnotetext{
${ }^{3}$ but we shall see in the sequel that this is far from being the case!
} 
3.3. The case of FFT-based schemes. This class of schemes became popular after the publication of [1, 2], mainly because treating the differential part of (2.6) by means of a discrete Fourier transform looked very much like being the best possible compromise in terms of meshing constraints. Indeed, in the linear case where (2.1) is supplemented with a potential $V(x)$ on its right-hand side, it was shown that the time-step $\Delta t$ could be chosen independent of $\varepsilon$ whereas the space discretization has to satisfy $\Delta x=\mathcal{O}(\varepsilon)$. This was already much better when compared to finitedifferences; moreover, the method is naturally $L^{2}$-conservative. In [2], these authors extended their "Fourier framework" to the weakly nonlinear Schrödinger equations of the form (2.6). However, and despite the fact we do believe these "FFT time-split schemes" realize the best numerical strategy in terms of gridding, we shall point out some shortcomings of the method in the next section.

3.4. A rigorous framework for FFT-based schemes. We present here a preliminary result about truncation errors in Lebesgue spaces for Fourier schemes; its proof follows directly from the Strichartz estimates on the torus due to J. Bourgain [5] (see also [6]), and from the study of FFT by M. Taylor [35, pp. 250-254]. Its derivation is not obvious though as it applies directly to widely-used schemes like the one recalled in the forthcoming section. We restrict our attention to the 1D free Schrödinger equation (2.1) with $\varepsilon=1$, and periodic boundary conditions: $x \in \mathbb{T}:=\mathbb{R} / 2 \pi \mathbb{Z}$.

Hence we start from

$$
i \partial_{t} \psi+\frac{1}{2} \partial_{x}^{2} \psi=0, \quad \psi(t=0, \cdot)=\zeta=\sum_{j \in \mathbb{Z}} \hat{\zeta}_{j} e^{i j x}, \quad x \in[0,2 \pi] .
$$

We have explicitly:

$$
\psi(t, x)=\sum_{j \in \mathbb{Z}} \hat{\zeta}_{j} e^{i j(x-j t / 2)} .
$$

In order to investigate the behavior of the FFT-scheme involving a finite even number $N \in 2 \mathbb{N}$ of modes, we introduce the Discrete Fourier Transform of a continuous function $f$ on $[0,2 \pi]$ as follows:

$$
f_{k}^{\#} \stackrel{\text { def }}{=} \frac{1}{N} \sum_{j=1}^{N} f(2 j \pi / N) e^{-i 2 \pi j k / N} .
$$

From [35, p. 252], we recall:

Lemma 3.2. If the continuous function $f$ has a convergent Fourier series, then:

$$
\forall k, \quad f_{k}^{\#}=\sum_{j \in \mathbb{Z}} \hat{f}_{k+j N} .
$$

Now, what the Fourier numerical scheme really computes is:

$$
\forall k \in\left\{-\frac{N}{2}, \frac{N}{2}\right\}, \quad \hat{\psi}_{k}^{\#}(t) \stackrel{\text { def }}{=} e^{-i k^{2} t / 2} \hat{\zeta}_{k}^{\#} .
$$

Thus the corresponding numerical solution $\psi^{\text {Num }}$ is built:

$$
\psi^{N u m}(t, x)=\sum_{k=-\frac{N}{2}}^{\frac{N}{2}} e^{i k(x-k t / 2)} \sum_{j \in \mathbb{Z}} \hat{\zeta}_{k+j N} .
$$


The question is therefore to study the discrepancy between $\psi$ and $\psi^{N u m}$ :

$$
\begin{aligned}
\psi^{N u m}(t, x)-\psi(t, x) & =\sum_{k=-\frac{N}{2}}^{\frac{N}{2}} e^{i k(x-k t / 2)} \sum_{j \in \mathbb{Z}} \hat{\zeta}_{k+j N}-\sum_{k \in \mathbb{Z}} \hat{\zeta}_{k} e^{i k(x-k t / 2)} \\
& =\sum_{j \in \mathbb{Z}}\left(\sum_{k=-\frac{N}{2}}^{\frac{N}{2}} e^{i k(x-k t / 2)} \hat{\zeta}_{k+j N}\right)-\sum_{k \in \mathbb{Z}} \hat{\zeta}_{k} e^{i k(x-k t / 2)} \\
& =\sum_{j \in \mathbb{Z}^{\star}}\left(\sum_{k=-\frac{N}{2}}^{\frac{N}{2}} e^{i k(x-k t / 2)} \hat{\zeta}_{k+j N}\right)+\sum_{|k|>\frac{N}{2}} \hat{\zeta}_{k} e^{i k(x-k t / 2)} \\
& \stackrel{\text { def }}{=} I+I I .
\end{aligned}
$$

The second term $I I$ in this last equality can be interpreted as a usual truncation error; it means that some high frequencies in the solution are lost when discretizing the problem on a finite grid. From [35, p. 253], we know that the modulus of this term can be controlled as follows:

$$
\sum_{|k|>\frac{N}{2}}\left|\hat{\zeta}_{k}\right| \leq \frac{1}{N^{m}}\|\zeta\|_{C^{1+m}},
$$

which is satisfactory provided the initial data $\zeta$ is smooth, e.g. $C^{2}(\mathbb{R})$. However, the first term $I$ is far more delicate and reveals the propagation of the errors coming from the DFT/FFT itself. It can be controlled thanks to the periodic Strichartz estimates proved by J. Bourgain. From [6, pp. 16-17], we recall:

Lemma 3.3. Given any complex-valued sequence $\left(a_{k}\right)_{k}$, the following estimates hold for the corresponding functions in $[0,2 \pi] \times[0,2 \pi]$ :

$$
\begin{aligned}
\left\|\sum_{k} a_{k} e^{i\left(k x-k^{2} t\right)}\right\|_{L^{4}(\mathbb{T} \times \mathbb{T})}^{2} & \leq C \sum_{k}\left|a_{k}\right|^{2}, \\
\left\|\sum_{|k| \leq N} a_{k} e^{i\left(k x-k^{2} t\right)}\right\|_{L^{6}(\mathbb{T} \times \mathbb{T})}^{2} & \leq C e^{2 \log N / \log \log N} \sum_{k}\left|a_{k}\right|^{2} .
\end{aligned}
$$

The second estimate looks more attractive as it "sees" the finite number of modes. We therefore deduce that the first term can be controlled in $L^{6}$ by means of:

$$
C \exp \left(\frac{\log N / 2}{\log \log N / 2}\right)\left(\sum_{j \in \mathbb{Z}^{\star},|k| \leq \frac{N}{2}}\left|\hat{\zeta}_{k+j N}\right|^{2}\right)^{\frac{1}{2}} .
$$

For instance, if $\zeta$ is a finite superposition of Fourier modes, then it is clear that this term cancels for $N$ large enough as $j \neq 0$ in the summation; obviously, the second term $I I$ vanishes too.

The general case isn't completely clear yet.

\section{EXPERIMENTS ON THE FOCAL POINT}

This section aims at visualizing the asymptotics previously recalled; namely we shall compare numerical approximations of (2.6) and (2.1) in 1D $(n=1)$ for various 
values of the parameters $\alpha$ and $\sigma$. The initial wave function is rather simple:

$$
v^{\varepsilon}(t=0, x)=u^{\varepsilon}(t=0, x)=\exp \left(-\left(2+\frac{i}{2 \varepsilon}\right)(x-\pi)^{2}\right), \quad x \in[0,2 \pi] .
$$

Numerical results have been obtained through the time-splitting FFT schemes recalled in the previous section; we used 1024 modes and fixed $\varepsilon=1 / 150$. It is convenient to observe results in $t=2$ since $\left|v^{\varepsilon}(t=2,).\right|=\left|v^{\varepsilon}(t=0,).\right|$.

4.1. Subcritical case. This case corresponds to $\sigma=2$ and $\alpha=2.5$; we expect to observe a decay of the absolute errors between $v^{\varepsilon}$ and $u^{\varepsilon}$ for values $\varepsilon \ll 1$. This is indeed the case, but Fig. 11 shows even a bit more, namely it compares pointwise the following quantities: (recall Lemma 2.1)

$$
\left\{\begin{array}{l}
\Re\left(v^{\varepsilon}(t=0, x) \exp \left(-i(x-\pi)^{2} / 2 \varepsilon\right)\right), \\
\Im\left(v^{\varepsilon}(t=2, x) \exp \left(i(x-\pi)^{2} / 2 \varepsilon\right)\right) \\
\Im\left(u^{\varepsilon}(t=2, x) \exp \left(i(x-\pi)^{2} / 2 \varepsilon\right)\right)
\end{array}\right.
$$

On the left in Fig. 1, we obviously observe that the absolute errors are slightly
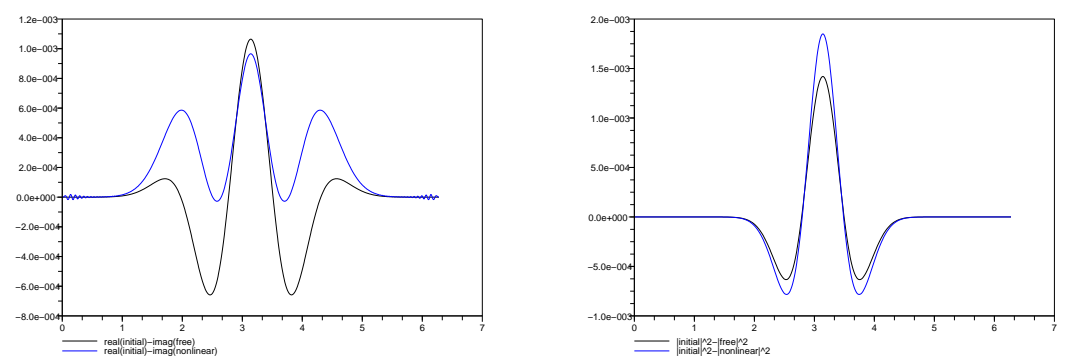

FiguRE 1. Absolute errors on the wave functions (left) and on the modulus (right) at $T=2$ with $\sigma=2$ and $\alpha=2.5$. (Subcritical case)

bigger when considering the solution of the nonlinear equation (2.6), $u^{\varepsilon}$. However, even for the free solution $v^{\varepsilon}$, one sees that the error doesn't vanish despite the fact no time-splitting algorithm is needed. As the way of discretizing the solution reveals itself important, we include here the corresponding SCILAB routine for the free equation:

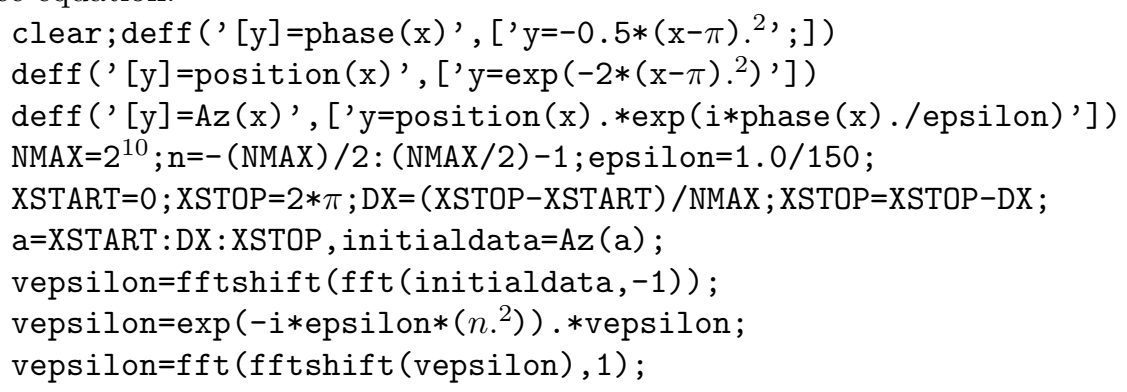

Clearly, its outcome is in agreement with Lemma 2.1 since $\varepsilon$ is already quite small. The Maslov index is visible, up to an error around $10^{-3}$ for 1024 Fourier modes. 
4.2. Critical case. We now put $\sigma=\alpha=2$ and the outcome is displayed on Fig. 2. we still compare the same quantities. Absolute errors on wave functions (left side) are much bigger for $u^{\varepsilon}$ in this case. In particular, no new frequencies appear in
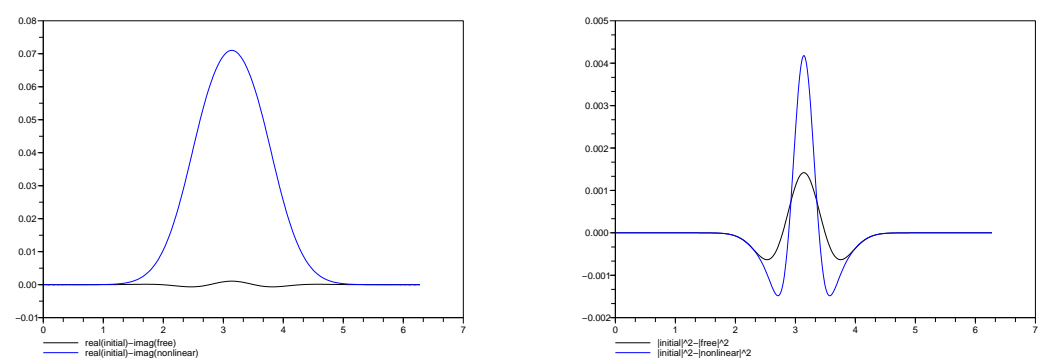

Figure 2. Same as Fig. 1 but $\sigma=2$ and $\alpha=2$. (Critical case)

the numerical solutions. The nonlinear effect boils down to a small change on the modulus of $u^{\varepsilon}$.

4.3. Supercritical case. We close this first series of tests by considering $\sigma=2$ and $\alpha=1.5$ as shown in Fig. 3 . Of course, as no pointwise convergence is expected in this case, absolute errors are even bigger for both wave functions (left side) and moduli (right side). Of course, the size of the error on the modulus is much bigger
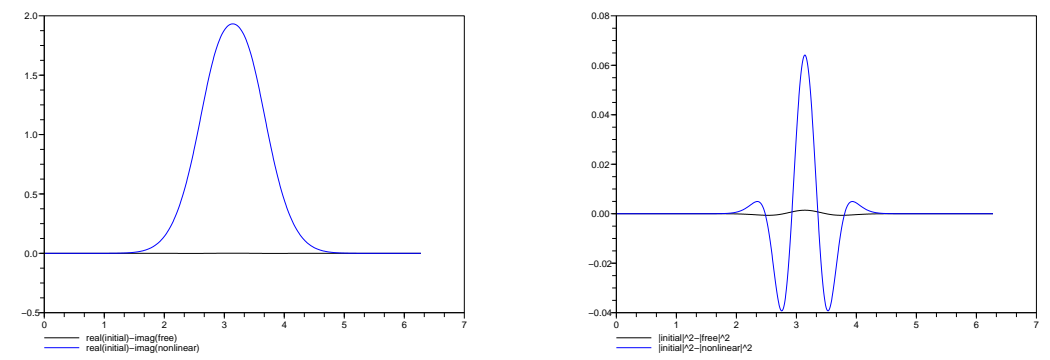

Figure 3. Same as Fig. 11, but $\sigma=2$ and $\alpha=1.5$. (Supercritical case)

too, and one should be extremely careful about the credit to give to the numerical simulations in the supercritical case. Indeed, this is a regime where a small error can be amplified at leading order (see [11, 12]).

\section{VisualizATION OF THE SCATTERING OPERATOR}

We aim now at illustrating the results on scattering theory through numerical computations still achieved through time-splitting FFT schemes. The algorithm we used for the approximation of the scattering operator is based on a nonlinear time-splitting routine flanked by two free evolution steps (implemented the way recalled in the previous section):

$$
S \psi \simeq U(-T) \circ U_{N L}(2 T) \circ U(-T) \psi, \quad T \gg 1,
$$


with $U, U_{N L}$ standing for the solution operators of equations 2.1) and (2.6) in 1-D with $\varepsilon=1$ respectively. We used $T=55$ in the computations hereafter.

As no small parameter $\varepsilon$ is present in the problem, one may think that no major obstacle exists in carrying out this program; this isn't correct as the free evolutions can (and do!) dramatically increase the size of the computational domain for large $T$. It is interesting to notice that, in case one wants to use FFT-based schemes, both the direct computation for small $\varepsilon$ and the scattering operator approximation lead to a "large computational domain difficulty": in the Fourier space for the first case, in the usual space for the second.

A way to understand the scattering operator is to visualize the average effects of the nonlinearities appearing in equations of the form

$$
i \partial_{t} u_{\lambda}+\frac{1}{2} \partial_{x}^{2} u_{\lambda}=\lambda\left|u_{\lambda}\right|^{2 \sigma} u_{\lambda}, \quad u_{\lambda}(t=0, x)=\exp \left(-5 x^{2}\right),
$$

for various values of $\sigma \geq 1$ and $\lambda$. Intuitively, as $\sigma$ increases, the nonlinearity becomes shorter range. Similarly, as $\lambda$ increases, the nonlinearity becomes stronger, and it should take a larger amount of time before we can consider it has become negligible. In all the tests we performed, it was somehow surprising to observe how fast the algorithm converges: one does not have to consider "very large" values of $T$ so that

$$
U(-T) \circ U_{N L}(2 T) \circ U(-T) \psi
$$

becomes stable and visually independent of $T$.

5.1. Quintic nonlinearity $(\sigma=2)$. The parameter $\lambda$ controls in some sense the strength of the nonlinearity ${ }^{4}$ inside (5.1), as can be seen on Fig. 4 . This figure displays the position density of the initial data, the scattered solution for $T=$ 55 and a "mixed state" $\tilde{u}_{\lambda}(t=0)=U(-T) U_{N L}(T) u_{\lambda}(t=0)$. As our timesplitting/FFT algorithm preserves only the $L^{2}$ norm, but not the Hamiltonian, we first restricted ourselves to moderate values of $\lambda \geq 0$ (defocusing case). However,
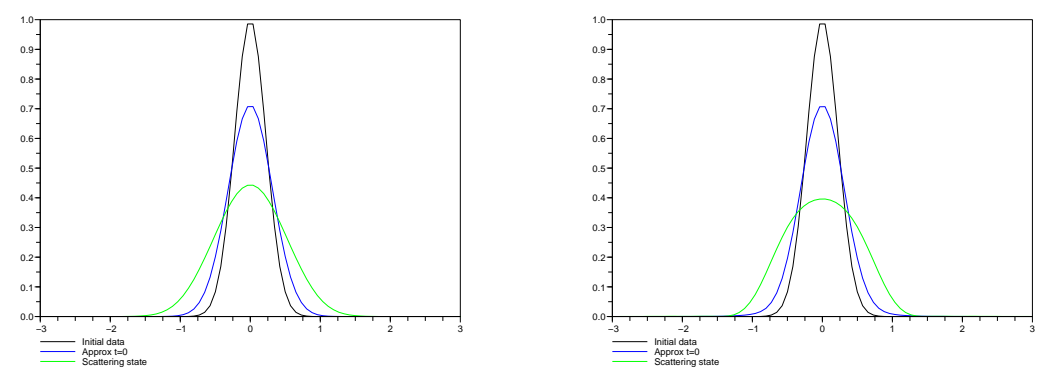

FiguRE 4. Initial data, numerical solution at $T=0$, scattering state for (5.1) with $\sigma=2$ and $\lambda=1$ (left), $\lambda=5$ (right)

as a numerical experiment, we wanted to display the outcome of our scheme for the stronger case $\lambda=25$ on Fig. 周: notice the change of shape in the scattered solution. Moreover, on this figure, we also tried to show what happens for $\lambda=-1$, that is to say for the focusing case despite there may be finite time blow-up (but there is scattering for small data). We checked that the energy associated to this

\footnotetext{
4 through the stiffness of the associated differential equation.
} 
data is (and remains) positive, a case where the virial identity, [14], does not imply blow-up. The computational domain for these runs was $[-100 \pi, 100 \pi]$ and $2^{13}-1$ Fourier modes were used.
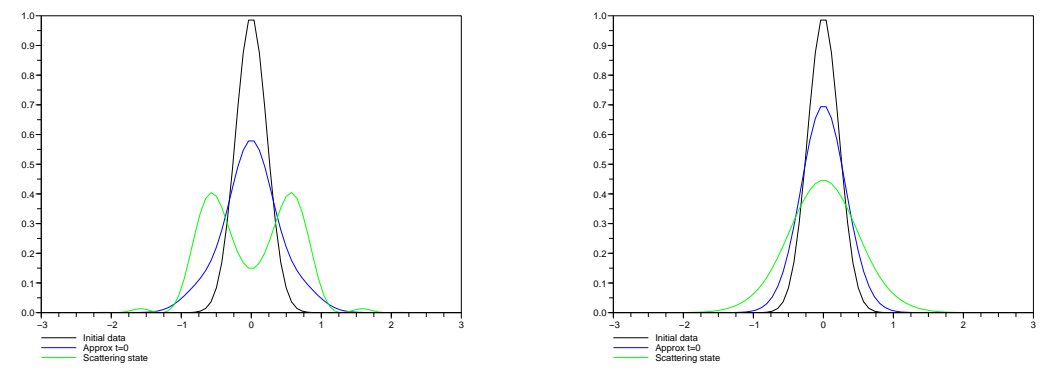

Figure 5. Same as Fig. 因, but $\lambda=25$ (left), $\lambda=-1$ (right)

5.2. Power $3(\sigma=1.5)$. Now let's observe the effects of lowering the $\sigma$ value while keeping other parameters equal, see Fig. 6. It is interesting to see that the change of shape appearing for $\lambda=25$ is stronger than in the preceding case. On the contrary, the increase of the numerical solution's support is slightly less important. This hints that increasing the $\sigma$ value tends to expand the support of the scattered
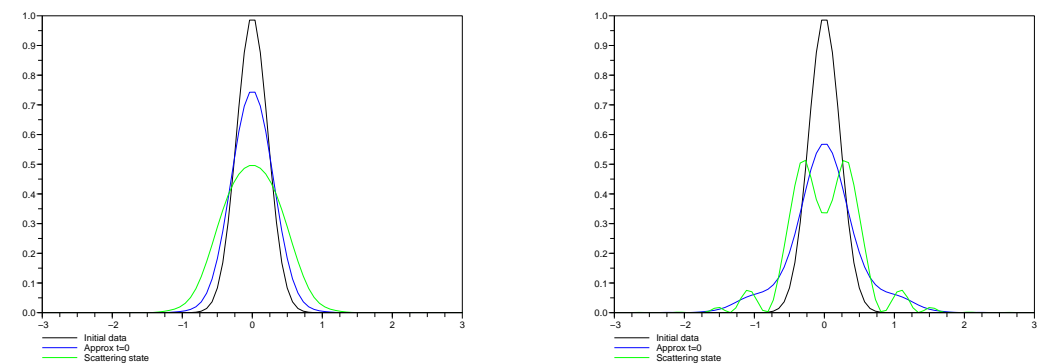

Figure 6. Same as Fig. A, but $\sigma=1.5$ and $\lambda=5$ (left), $\lambda=25$ (right)

solution whereas increasing the $\lambda \geq 0$ value (defocusing case) leads to an oscillatory behavior. However, we stress that since the energy,

$$
E(t):=\frac{1}{2}\left\|\partial_{x} u^{\lambda}(t)\right\|_{L^{2}}^{2}+\frac{\lambda}{\sigma+1}\left\|u^{\lambda}(t)\right\|_{L^{2 \sigma+2}}^{2 \sigma+2}=E(0),
$$

of the numerical solution changes more with a bigger $\lambda$ (its mass being always kept constant), these oscillations might be spurious. We actually don't know how this fact can be decided; our profiles have been checked to be stable on a finer grid.

5.3. Power $6(\sigma=3)$. In order to get some numerical evidence about the dependence of the scattered solution on $\sigma$, we display on Fig. 目 the outcome for $\sigma=3$. It is quite clear that the scattered solutions for both values of $\lambda$ are less peaked. Their support is bigger and the oscillations for $\lambda=25$ are weaker, their frequency remained the same though. This agrees with the behavior we sketched in the preceding subsection as $\sigma$ and $\lambda$ vary. 

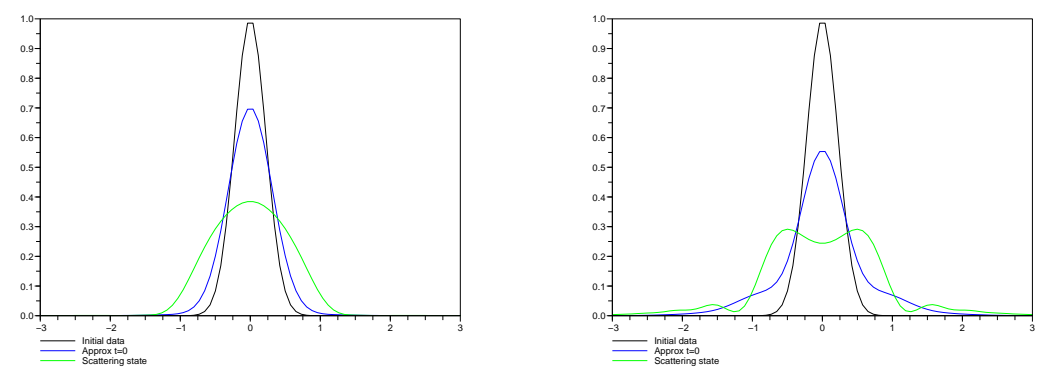

Figure 7. Same as Fig. 目, but $\sigma=3$ and $\lambda=5$ (left), $\lambda=25$ (right)

\section{EXPERIMENTS ON A CUSP CAUSTIC}

Let us now go back to comparing the quadratic observables generated by numerical approximation of equations (2.6) and (2.1) endowed with a small parameter $\varepsilon$ in 1-D. In this section we fixed $\varepsilon=1 / 150$. Figure 8 displays the position density of the initial data for both equations, i.e.

$$
u^{\varepsilon}(t=0, x)=v^{\varepsilon}(t=0, x)=\exp \left(-2(x-\pi)^{2}-i \cos (x) / \varepsilon\right), \quad x \in[0,2 \pi],
$$

together with the position density of the numerical approximations of (2.6), (2.1) in $T=3.5$. The point here is to investigate what happens for the case of such a self-interfering Gaussian pulse, since no scattering theory is known for this problem. What we would like to check is whether the theoretical results on the focus point recalled and visualized in the preceding sections can be thought of as a guideline for this more complex case involving a non-trivial caustic. We shall observe position densities for the unique value of $\sigma=4$ as a similar behavior has been seen to hold for different nonlinearities with convenient values of $\alpha$. 4095 Fourier modes have been used in order to produce these results.

6.1. Subcritical picture: $\alpha=4$. This case could be referred to as subcritical since it is noticeable on the top of Fig. 8 that the free and the nonlinear numerical solutions do agree for this reasonably small value of $\varepsilon$. In particular, the frequencies of oscillations are identical. This is very similar compared to the behavior investigated in [8].

6.2. Critical picture: $\alpha=3$. The parameter $\alpha$ is now in a "critical range" as we observe that both solutions differ much more, but the frequency of the oscillations looks like being still the same in both cases. In order to establish this fact, we display on the left of Fig.9 the FFT of the position densities: a peak at the same frequency is clearly noticeable. The nonlinear effect manifests itself through a change of order zero in the moduli, as we already observed on the right side of Fig. 2; notice also the similarity with the scattering state shown on Fig. 5 (right). This does agree with the $\alpha_{c}$ value derived in Section 2.2

6.3. Supercritical picture: $\alpha=2$. In this last case, there is no similarity no more between the approximate solutions of (2.6), (2.1), as seen on both Fig. 8 and 9. Especially, the right side of Fig. 9 reveals that new frequencies show up inside the position density of the nonlinear solution. We have therefore a change of order 

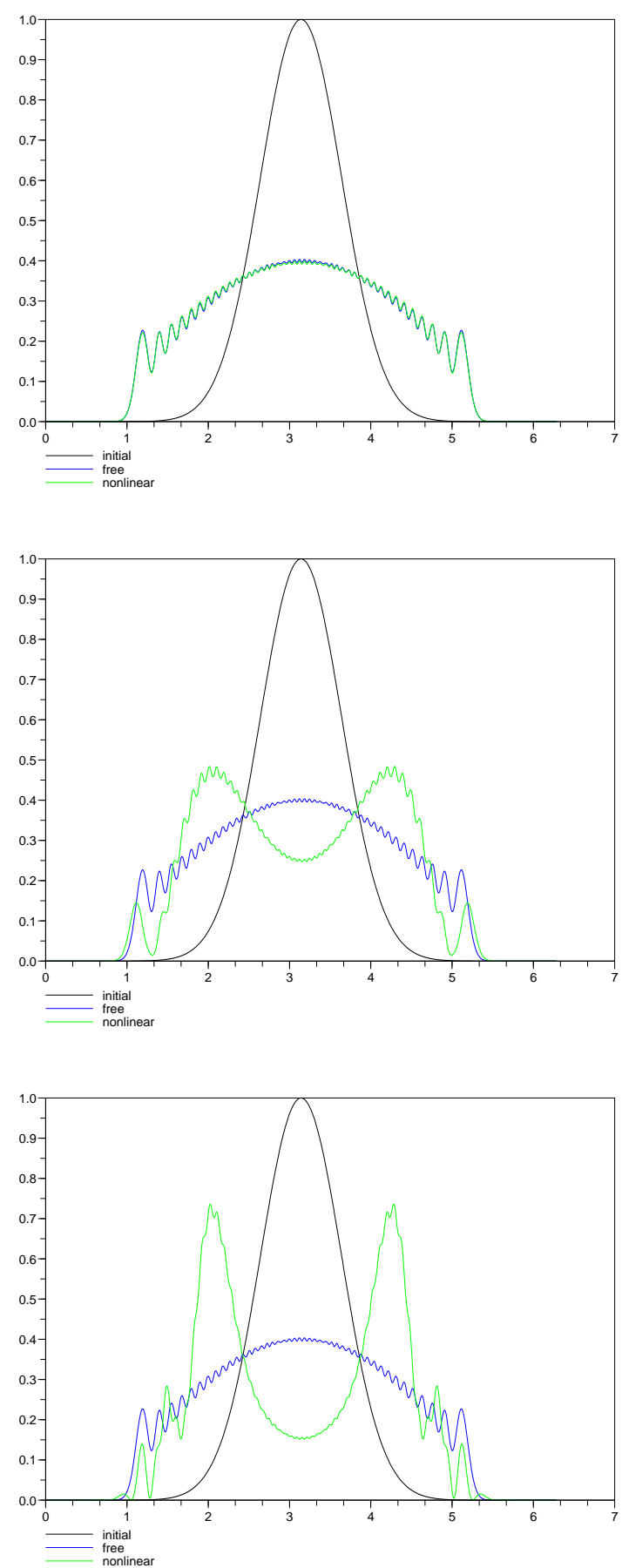

Figure 8. Position densities in the cusp caustic: $\alpha=4,3,2$ (top to bottom) 

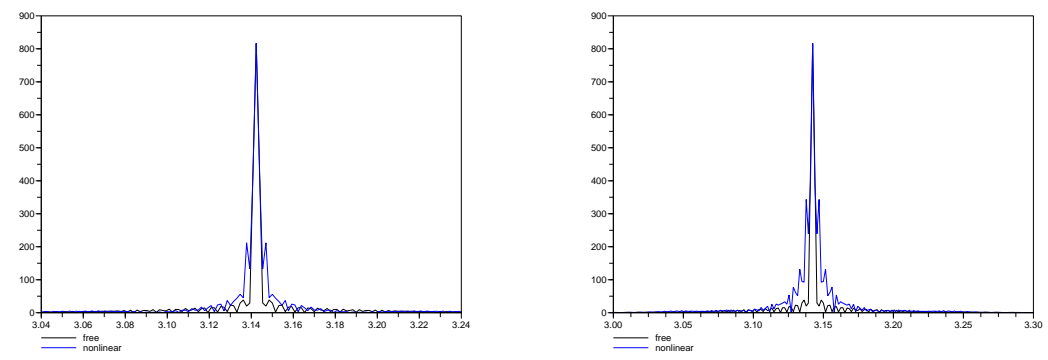

FiguRE 9. Fourier transforms of the position densities with $\alpha=3$ (left), $\alpha=2$ (right)

zero in the moduli and in the frequency. This is of course reminiscent of Fig. 3 in which a frequency doubling seems to show up in the supercritical regime.

\section{Conclusion}

We have presented the semi-classical limit for the nonlinear Schrödinger equation in the presence of a caustic. When the caustic is reduced to a point, the numerical experiments are in good agreement with the analytical results as far as the notion of criticality is concerned. However in the critical case, described by a nonlinear scattering operator, the leading order nonlinear effects are rather hard to visualize in the semi-classical limit. This is why we simulated the scattering operator in a separate way.

Our numerical tests give encouraging evidence of new phenomena concerning the phase of the wave in the supercritical case when a focal point is formed (appearance of new frequencies). In the presence of a cusp caustic, the numerical experiments are in good agreement with the heuristic arguments that we presented here, for which no rigorous justification is available so far.

\section{REFERENCES}

[1] W. Bao, S. Jin, and P. A. Markowich, On time-splitting spectral approximations for the Schrödinger equation in the semiclassical regime, J. Comput. Phys., 175 (2002), no. 2, 487524.

[2] W. Bao, S. Jin, and P. A. Markowich, Numerical study of time-splitting spectral discretizations of nonlinear Schrödinger equations in the semiclassical regimes, SIAM J. Sci. Comput., 25 (2003), no. 1, 27-64.

[3] J. E. Barab, Nonexistence of asymptotically free solutions for nonlinear Schrödinger equation, J. Math. Phys., 25 (1984), 3270-3273.

[4] C. Besse, B. Bidégaray, and S. Descombes, Order estimates in time of splitting methods for the nonlinear Schrödinger equation, SIAM J. Numer. Anal., 40 (2002), no. 1, 26-40.

[5] J. Bourgain, Fourier restriction phenomena for certain lattice subsets and applications to nonlinear evolution equations. I. Schrödinger equations, Geom. and Funct. Anal., 3 (1993), $107-156$.

[6] J. Bourgain, Nonlinear Schrödinger equations, in: Hyperbolic equations and frequency interactions, eds. L. Cafarelli and W. E. IAS/Park City vol. 5.

[7] N. Burq and M. Zworski, Instability for the semiclassical non-linear Schrödinger equation, Comm. Math. Phys. 260 (2005), no. 1, 45-58.

[8] R. Carles, Geometric optics with caustic crossing for some nonlinear Schrödinger equations, Indiana Univ. Math. J., 49 (2000), pp. 475-551. 
[9] R. Carles, Remarques sur les mesures de Wigner, C. R. Acad. Sci. Paris, t. 332, Série I, 332 (2001), pp. 981-984.

[10] R. Carles, Semi-classical Schrödinger equations with harmonic potential and nonlinear perturbation, Ann. Inst. H. Poincaré Anal. Non Linéaire, 20 (2003), no. 3, 501-542.

[11] R. Carles, Cascade of phase shifts for nonlinear Schrödinger equations, J. Hyperbolic Differ. Equ., (2007). To appear.

[12] R. Carles, Geometric optics and instability for semi-classical Schrödinger equations, Arch. Ration. Mech. Anal. 183 (2007), no. 3, 525-553.

[13] R. Carles, WKB analysis for nonlinear Schrödinger equations with potential, Comm. Math. Phys. 269 (2007), no. 1, 195-221.

[14] T. Cazenave, Semilinear Schrödinger equations, vol. 10 of Courant Lecture Notes in Mathematics, New York University Courant Institute of Mathematical Sciences, New York, 2003.

[15] T. Cazenave and F. Weissler, Rapidly decaying solutions of the nonlinear Schrödinger equation, Comm. Math. Phys. 147 (1992), 75-100.

[16] F. Dalfovo, S. Giorgini, L. P. Pitaevskii, and S. Stringari, Theory of Bose-Einstein condensation in trapped gases, Rev. Mod. Phys., 71 (1999), no. 3, 463-512.

[17] J. J. Duistermaat, Oscillatory integrals, Lagrange immersions and unfolding of singularities, Comm. Pure Appl. Math. 27 (1974), 207-281.

[18] A. Durán and J. M. Sanz-Serna, The numerical integration of relative equilibrium solutions. The nonlinear Schrödinger equation, IMA J. Numer. Anal. 20 (2000), 235-261.

[19] J. Ginibre, An introduction to nonlinear Schrödinger equations, in: Nonlinear waves (Sapporo, 1995), eds. R. Agemi, Y. Giga, and T. Ozawa, GAKUTO International Series, Math. Sciences and Appl., Gakkōtosho, Tokyo, 1997, pp. 85-133.

[20] J. Ginibre, T. Ozawa, and G. Velo, On the existence of the wave operators for a class of nonlinear Schrödinger equations, Ann. IHP (Physique Théorique) 60 (1994), 211-239.

[21] J. Ginibre and G. Velo, On a class of nonlinear Schrödinger equations. II Scattering theory, general case, J. Funct. Anal. 32 (1979), 33-71.

[22] L. Gosse, A case study on the reliability of multiphase WKB approximation for the onedimensional Schrödinger equation, in: Numerical methods for hyperbolic and kinetic problems, vol. 7 of IRMA Lect. Math. Theor. Phys., Eur. Math. Soc., Zürich, 2005, pp. 131-141.

[23] L. Gosse, S. Jin, and X. Li, Two moment systems for computing multiphase semiclassical limits of the Schrödinger equation, Math. Models Methods Appl. Sci. 13 (2003), no. 12, $1689-1723$.

[24] L. Hörmander, The analysis of linear partial differential operators, Springer-Verlag, Berlin, 1994.

[25] J. Hunter and J. Keller, Caustics of nonlinear waves, Wave motion 9 (1987), 429-443.

[26] J.-L. Joly, G. Métivier, and J. Rauch, Focusing at a point and absorption of nonlinear oscillations, Trans. Amer. Math. Soc. 347 (1995), no. 10, 3921-3969.

[27] J.-L. Joly, G. Métivier, and J. Rauch, Caustics for dissipative semilinear oscillations, Mem. Amer. Math. Soc. 144 (2000), no. 685, pp. viii+72.

[28] G. T. Kossioris, Formation of singularities for viscosity solutions of Hamilton-Jacobi equations in one space variable, Comm. Partial Differential Equations 18 (1993), 747-770.

[29] D. Ludwig, Uniform asymptotic expansions at a caustic, Comm. Pure Appl. Math. 19 (1966), $215-250$.

[30] P. A. Markowich, P. Pietra, and C. Pohl, Numerical approximation of quadratic observables of Schrödinger-type equations in the semi-classical limit, Numer. Math. 81 (1999), 595-630.

[31] K. Nakanishi and T. Ozawa, Remarks on scattering for nonlinear Schrödinger equations, NoDEA Nonlinear Differential Equations Appl. 9 (2002), no. 1, 45-68.

[32] L. Pitaevskii and S. Stringari, Bose-Einstein condensation, vol. 116 of International Series of Monographs on Physics, The Clarendon Press Oxford University Press, Oxford, 2003.

[33] W. A. Strauss, Nonlinear scattering theory, in: Scattering theory in mathematical physics, eds. J. Lavita and J. P. Marchand (Reidel, 1974).

[34] W. A. Strauss, Nonlinear scattering theory at low energy, J. Funct. Anal. 41 (1981), 110-133.

[35] M. Taylor, Partial Differential Equations, Vol. I, Springer, New York, 1996. 
(R. Carles) Institut CNRS Pauli, Wolfgang Pauli Institute c/o Fak. f. Mathematik., Univ. Wien, UZA 4, Nordbergstr. 15, A-1090 Wien, Austria ${ }^{5}$

E-mail address: Remi.Carles@math.cnrs.fr

(L. Gosse) Istituto per le Applicazioni del Calcolo (sezione di Bari), Via G. Amendola 122, 70126 Bari, Italy

E-mail address: 1.gosse@ba.iac.cnr.it

${ }^{5}$ Permanent address: Univ. Montpellier 2, UMR CNRS 5149, Mathématiques, CC 051, Place Eugène Bataillon, 34095 Montpellier cedex 5, France. 